\title{
CRASH RISK; EYE MOVEMENT AS INDICES FOR DUAL TASK DRIVING WORKLOAD
}

\author{
Julie J. Kang, Zheng Bian \& George J. Andersen \\ Department of Psychology \\ Visual Cognition and Performance Lab \\ University of California, Riverside \\ Email: andersen@ucr.edu
}

\begin{abstract}
Summary: The goal of the present study was to examine eye movements as a function of dual task difficulty while driving. Two tasks were examined: maintaining a predetermined distance while car following and detecting a light change. Task demands were manipulated by varying the amplitude of lead vehicle's (LV) speed change and increasing the average LV speed. As task demands increased, the number of saccades decreased. There was no significant difference in number of fixations, fixation duration, number of eye blinks, or pupil size. While car following performance did not change, drivers were more accurate at the light detection task at the $100 \%$ amplitude condition verses the $120 \%$.
\end{abstract}

\section{INTRODUCTION}

Driving requires the operator to simultaneously steer their vehicle, scan for potential dangers, while navigating a safe route. While drivers are generally quite capable of performing multiple tasks, studies have shown that performance suffers as attentional demands increase. Drivers engaged in complex tasks are three times more likely to be involved in an automobile crash compared to drivers performing simple tasks (Wang, Knipling, Goodman, 1995). The National Highway Traffic Safety Administration (NHTSA) cites driver distraction as responsible for approximately $25 \%$ - $30 \%$ of all police-reported collisions.

Drivers performing complex tasks may be less efficient at detecting hazards. In this paper, two potential explanations are presented for why drivers miss hazards. The first explanation is that drivers were "looking but failed to see" the critical target (Crundall, Shenton, Underwood, 2004). It has been reported that increased workload is associated with a reduction in the useful field of view (UFOV) (Miura, 1986). The UFOV is the visual area that a driver can rapidly process information (Owsley, Ball, McGwin, 1998). It is hypothesized that drivers make more saccades to sample additional information to compensate for a smaller UFOV. The increased sampling rate decreases the amount of information that is processed within a single fixation, accounting for the "look but failed to see" errors in driving.

The second explanation is that drivers experience cognitive tunneling under higher workload conditions (Martens \& van Winsum, 2000). Cognitive tunneling occurs when the operator focuses attention to one area of interest to the exclusion of information presented outside this region (Thomas, Wickens, 2001). Thus, drivers may fail to detect hazards due to prioritizing only one aspect of the scene. 
The paper will compare two competing hypotheses regarding eye movements and attention. One hypothesis is the "look but failed to see" hypothesis. According to this hypothesis, the UFOV is reduced as a function of workload. As a result performance for the light detection task should decrease more for peripherally located lights than for centrally located lights, particularly when the car following task becomes more difficult (i.e. higher level of LV amplitude or higher LV velocity). As a result of a reduction in the UFOV, the number of saccade will increase between critical targets in the driving scene (i.e. lead vehicle and traffic lights). The second hypothesis is that the number of saccades will decrease as task demands increase allowing the driver to focus attention on a single task. We will refer to this hypothesis as the cognitive tunneling hypothesis. According to this hypothesis drivers will increase fixation at regions of interest and decrease scans to peripheral areas when performing more difficult tasks.

To compare these two hypotheses we examined performance on a car following and light detection task. For the car following task drivers were instructed to maintain a 21 meter following distance (presented during the first $5 \mathrm{sec}$ of each trial) behind the LV. The drivers followed a LV that varied its velocity according to a sum of 3 prime frequency sinusoids $(0.033$, $0.083,0.117 \mathrm{~Hz}$ ). The car following task's level of difficulty was manipulated by varying (1) average LV speed (60 and $100 \mathrm{kmph}$ ) and (2) amplitudes of the frequencies in the LV speed profile (100\% and 120\%). The corresponding sine waves to the amplitude of LV's speed change $100 \%$ and $120 \%$ were $9.722,3.889$, and $2.778 \mathrm{kmph}$ or $11.66,4.65$, or $3.34 \mathrm{kmph}$, respectively.

On each trial the driver would drive past 24 light arrays positioned horizontally between two buildings (see Figure 1). Each array was composed of 21 red and green lights (in a randomized pattern). One light per array changed colors at the 3rd, 6th, 9th position (relative to the array's center). The light changed at randomized, preset distances $(24,36,48,60$ meters) between the driver's vehicle and the array. Drivers were instructed to indicate whether a left or right light changed as quickly and accurately as possible. The distance and position of light change was randomized so that the onset and location of the change was not predictable. The position of the light change was used as an approximate indication of whether the size of the UFOV changed as a function of car following difficulty.

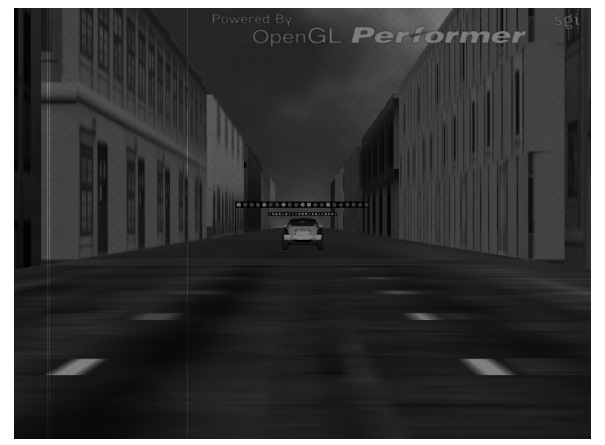

Figure 1. Screen shot of the lead vehicle and light arrays in the driving scene.

We used both local measures and global measures to assess car following performance. Local measures included control gain, phase angle, and squared coherency for the three frequencies in the LV speed profile. Global measures included average headway distance, headway distance 
variance, and RMS error of car following performance. For the light detection task we examined accuracy and reaction times. Ocular indices of workload were assessed by examining the number of saccades, duration of saccades, number of fixations, duration of fixations, pupil size, and number of blinks.

\section{EXPERIMENT}

\section{Methods}

Drivers. Eight college students (mean age $=22$ ) were recruited and paid for their participation. Prior to the experiment, all drivers submitted to visual, attentional, and cognitive tests including useful field of view (UFOV), Snellen acuity, contrast sensitivity, WAIS-KBIT, and colorblindness. All reported normal or corrected-to-normal vision and were currently licensed drivers.

Design. The independent variables were LV's speed (60 and $100 \mathrm{kmph}$ ), amplitude of LV's speed change (100\% or $120 \%)$, and distance of light change $(24,36,48,60$ meters).

Apparatus. The displays were presented on Dell 670 Workstation desktop computer. A Thrustmaster Formula T2 steering system with acceleration and brake pedals, was used for closed loop control of the simulator. The foot pedal and a BG systems serial box (an analog to digital converter system) were used to produce closed loop control that was updated at $36 \mathrm{~Hz}$. The displays were presented on a $25.8 \mathrm{deg}$ by $34.7 \mathrm{deg}$ display. The approximate diagonal measurement of the screen was 23.1" with a 4:3 ratio. The viewing distance was 36 ". Eye movements were measured at $250 \mathrm{~Hz}$ using an Eyelink II eye tracker.

Driving Scenario. The roadway consisted of three traffic lanes with the driver and lead vehicle located in the center lane (figure 1). Lane width was 3.8 meters.

A black and white gravel texture pattern was used to simulate asphalt. Dashed lines ( 2 meters in length positioned every 2 meters along the roadway) were used to simulate lane markers. The city buildings and LV were produced by digitally photographing real buildings and vehicle and using the digital images as texture maps for the roadway scenes. The images were digitally altered to increase the realism of the simulator scene (e.g., remove specular highlights, add shading) and were scaled to be appropriate with the geometry of the simulation. The lead vehicle (LV) was a white colored sedan located at a headway distance of 21 meters.

Procedure. Drivers were instructed to perform both car following and light detection tasks. At the beginning 5 seconds of each trial run, the driver's vehicle maintained a constant distance of 21 meters behind the LV with no control input to establish a perception of the desired following distance. The three sinusoids used to create the velocity for the LV were out of phase with one another. The initial phase of the high and middle frequency was selected randomly with the phase value of the low frequency to produce a sum of zero. This manipulation ensured that the velocity profile of the lead vehicle would vary from trial to trial with a smooth speed transition following the period of constant speed. 
The driver also performed a light detection task. Button responses were entered on the steering wheel. The dependent measures for light detection were percent correct and response times.

\section{Results}

To examine light detection performance, we conducted a four-way, within-subject ANOVA with repeated measures. The independent variables were LV's speed (60 and $100 \mathrm{kmph}$ ), amplitude of LV's speed change $(100 \%$ or $120 \%)$, light position $\left(3^{\text {rd }}, 6^{\text {th }}, 9^{\text {th }}\right)$ and distance of light change $(24$, $36,48,60$ meters). The average accuracy (percent correct) and reaction time (msec) were recorded and analyzed for each subject in each condition.

To examine car following performance, we conducted a three-way, within-subject ANOVA with repeated measures. The independent variables were LV's speed (60 and $100 \mathrm{kmph}$ ), amplitude of LV's speed change $(100 \%$ or $120 \%)$, frequency of speed change $(0.033,0.083,0.117 \mathrm{~Hz})$. Car following performance was assessed by deriving average control gain, phase angle, squared coherency for the three frequencies in the LV speed profile, and average headway distance, headway distance variance, and RMS error of car following performance for each subject in each condition.

To examine eye movements, we conducted a four-way, within-subject ANOVA with repeated measures. The independent variables were LV's speed (60 and $100 \mathrm{kmph}$ ), amplitude of LV's speed change $(100 \%$ or $120 \%)$, light position $\left(3^{\text {rd }}, 6^{\text {th }}, 9^{\text {th }}\right)$ and distance of light change $(24,36$, 48, 60 meters). Eye movement patters were assessed by determining the average number of saccades, duration of saccades, number of fixations, duration of fixations, pupil size, and number of blinks for each subject in each condition.

\section{Average LV Speed}

There was a main effect of average LV's speed for response time, $F(1,7)=8.518, p=.022$. Drivers were slower to respond to the light change $(M=792 \mathrm{~ms})$ when the LV's average speed was 60 $\mathrm{kmph}$ compared to $100 \mathrm{kph}(\mathrm{M}=605 \mathrm{~ms})$. There was also a main effect of average LV's speed for accuracy, $\mathrm{F}(1,7)=22.837, \mathrm{p}=.002$. Drivers were more accurate to the light detection task $(\mathrm{M}=$ $89 \%$ ) when the LV's average speed was $60 \mathrm{kmph}$ compared to $100 \mathrm{kmph}(\mathrm{M}=84 \%)$. Finally, there was a main effect for average LV's speed for phase angle, $F(1,7)=17.744, p=.004$. Drivers had less response sensitivity to changes in the LV's speed $(\mathrm{M}=-.54$ cycle of a sine wave) when the LV's average speed was $60 \mathrm{kmph}$ compared $100 \mathrm{kmph}(\mathrm{M}=-.66$ cycles per sine wave $)$.

\section{Amplitude of LV's Speed}

There was a main effect of amplitude of LV's speed changes for response time, $F(1,7)=6.0842$, $\mathrm{p}=.043$. Drivers responded more quickly to the light change $(\mathrm{M}=853 \mathrm{~ms})$ when the LV's amplitude of speed change was $100 \%$ compared to $120 \%(\mathrm{M}=879 \mathrm{~ms})$. There was also a main effect of amplitude for accuracy, $\mathrm{F}(1,7)=6.08, \mathrm{p}<.05$. Drivers were less accurate in the light detection task ( $\mathrm{M}=85.9 \%)$ when the amplitude of the LV's speed change was $100 \%$ as compared to $120 \%(\mathrm{M}=87.2 \%)$. Finally, there was a main effect of amplitude for the number of saccades. Driver made less saccades when the amplitude of the LV increased from $100 \%(\mathrm{M}=443$ saccades) to $120 \%(M=419$ saccades $), F(1,7)=7.24, p<.05$. The computed Spearman's correlation coefficient of the number of saccades and accuracy is $r=-.21[\mathrm{p}<0.002,95 \% \mathrm{CI}]$. 
However, there were no significant effects for duration of saccades, number of fixations, fixation duration, number of blinks, or pupil size.

\section{Light Position}

There was a main effect of light position on the driver's response time to light changes, $\mathrm{F}(2$, $14)=3.99, p=.042$. Drivers were faster at responding to light changes in the $9^{\text {th }}$ position $(\mathrm{M}=840$ $\mathrm{ms})$ compared to the $3^{\text {rd }}$ position $(\mathrm{M}=909 \mathrm{~ms})$. There was also a main effect of light position for accuracy, $\mathrm{F}(1,7)=22.837, \mathrm{p}=.002$. Drivers were less accurate as the distance from central fixation increased, $(\mathrm{M}=90 \%)$ for the $3^{\text {rd }}$ position verses $(\mathrm{M}=84 \%)$ for the $9^{\text {th }}$ position, $\mathrm{p}=.03$. Finally, there was an interaction of light position and amplitude of LV's speed change on response time, $\mathrm{F}(2,14)=6.1, \mathrm{p}=.012$. There was no significant difference in response times when the average LV velocity was 60 or $100 \mathrm{kmph}$, at the $3^{\text {rd }}$ and $6^{\text {th }}$ light position. At the $9^{\text {th }}$ position, response times increased when the LV's average speed was $60 \mathrm{kmph}(\mathrm{M}=768 \mathrm{~ms})$ but remained the same as the $3^{\text {rd }}$ and $6^{\text {th }}$ position when the LV's average speed was $100 \mathrm{kmph}(\mathrm{M}=703 \mathrm{~ms})$. There was no significant interaction of light position and amplitude of LV's change in speed on accuracy, $\mathrm{F}(2$, $14)=.570, \mathrm{p}=.58$. It is important to note that this is only an approximate measure of UFOV since the drivers were performing a continuous car following task and not a discrete identification task.

\section{DISCUSSION}

The present study examined eye-movement patterns as a function of dual task difficulty within the context of driving. Specifically, we were interested in whether increased dual task difficulty resulted in errors of "looking but failed to see" or cognitive tunneling. First, we examined whether the extent of UFOV changed as a function of task difficulty (average LV speed or amplitude of LV speed change). The UFOV was indirectly measured by examining light detection performance by light position $\left(3^{\text {rd }}, 6^{\text {th }}, 9^{\text {th }}\right)$ and car following difficulty. The assumption is that the UFOV will shrink in the more challenging car following conditions. This would be indicated by a systematic decrease in performance with increased distance from the central light position. While the results did indicate that drivers were faster but less accurate at detecting light changes at the most peripheral positions, the results did not support a change in UFOV with increased task difficulty. Drivers were more accurate at detecting a light change when the car following task was more difficult (higher LV amplitudes).

A simple explanation is that the drivers are experiencing cognitive tunneling (i.e., spending longer fixation durations on the light array than on the lead vehicle). However, there are two results that conflict with this interpretation. First, the increase in accuracy did not occur as a result of performing the car following task. If the drivers were selectively prioritizing the light detection task over the car following task, then this would be revealed by a cost in the driving measures. Secondly, there was no significant effect of fixations on amplitudes. The improved accuracy on the light detection cannot be accounted for by longer fixations.

Instead the results suggest that the greater performance may be due to the amount of visual information processed. Since visual information is not processed during a saccade, it was hypothesized that decreasing the number of saccades will result in better performance. Indeed, the highest accuracy occurred when drivers were making the least number of saccades. The results indicated that drivers reduced the number of saccades at higher LV amplitude conditions. 
The results seem to indicate that the drivers adapted a different scan strategy depending on the difficulty of the car following task. Specifically, drivers made more saccades when car following was less demanding (e.g. lower amplitudes of LV speed change) and fewer saccades when car following was more demanding. A correlation of $r=-0.21$ indicates that corresponding increase in the number of saccades for light detection task as for higher amplitude conditions (i.e. more difficult car following task).

One possible explanation for this result is that initiating fewer saccades may optimize performance as workload increased. Several studies report that decreased eye movements are associated with better performance in non-driving domains, such as complex video games (Shapiro \& Raymond, 1989), air traffic control (Stein, 1992), and general visual search tasks (Boot, Kramer, Becic, Wiegmann, Kubose, 2006). Boot and colleagues reported that the type of scan strategy employed was predictive of the level of performance on a detection task. Specifically, participants who were instructed to scan the scene performed the worst while participants who were instructed to minimize eye movements performed the best. In the present study, the drivers were not given specific instructions about which scan strategy to adopt while viewing the driving scene. However, it appears as though drivers selectively adapted to make fewer saccades as the car following task became more difficult. An important issue for future research will be to examine car following performance with a greater change in LV speed by increasing the amplitude of the complex sine wave function.

Surprisingly, there was no significant difference on car following performance when the LV's speed increased or amplitude of LV's speed change increased. One explanation is that speed and amplitude levels selected did not challenge the drivers. A fundamental assumption of attention studies is that there will be a performance cost by increasing the difficulty of the task. One problem with this assumption is that the operator's performance may not necessarily reflect the difficulty of the task. For example, if the initial task does not require much effort, then it is possible that the participant has a reserve of attentional resources to draw upon as the task became more challenging. Thus, when effort is increased as the task becomes more challenging, performance on the more difficult task could be the same or better than on the less difficult task. Therefore, task difficulty would not be indicated by diminished performance since the challenging task is met with additional attentional effort.

In summary, while the results did not indicate a change in the extent of the attended region (UFOV), the results did suggest that drivers reduced the number of saccades when the car following task becomes more challenging. The reduction in the number of saccades may be helpful in allowing the driver to process more visual information. We interpreted the reduction of saccades as reflecting an increase in concentration in response to increased task difficulty.

\section{REFERENCES}

Boot, W. R.; Kramer, A. F.; Becic, E.; Wiegmann, D.A.; Kubose, T. (2006). Detecting transient changes in dynamic displays: The more you look, the less you see. Human Factors, 48, 759773.

Crundall, D.; Shenton, C.; Underwood, G.; (2004). Eye movements during intentional car following. Perception, 33, 975 - 986. 
Martens, M. H.; van Winsum, W. Measuring distraction: the Peripheral Detection Task. Proceedings NHTSA, 2000

Miura, T. (1986). Coping with situational demands: A study of eye movements and peripheral vision performance. In A.G. Gale, I.D. Brown, C.M. Haslegrave, P. Smith \& S. Taylor (eds.), Proceedings of Vision in Vehicles, (p. 205-216). Amsterdam: Elsevier, North Holland.

Owsley, C., Ball, K., McGwin, G. Jr. \& et al. (1998). Visual processing impairment and risk of motor vehicle crash among older adults. JAMA: Journal of the American Medical Association. 1998, 279: 1083-1088.

Rantanen, E. \& Goldberg, J. (1999). The effects of mental workload on the visual field shape and size. Ergonomics, 42, $816-834$.

Shapiro \& Raymond, (1989). Training the efficient ocularmotor strategies enhances skill acquisition. Acta Psychologica. 71, 2170241.

Stein, E.S. (1992). Air traffic control visual scanning. (DOT/FAA/CT-TN92/16). Atlantic City, NJ: Department of Transportation, Federal Aviation Administration Technical Center.

Wang, J. S.; Knipling, R. R.; Goodman, M. J., The role of driver inateiont in crashes: New statistics from the 1995 Crashworthiness Data System. 40th Annual Proceedings of the Association of the Advancement of Automotive Medicine., 377-392. Vancouver, British Columbia.

Wierwille, W.W., Hulse, M.C., Fischer, T.J., \& Dingus, T.A. (1988). Stategic use of vision resources by the driver while navigating with an in-car navigation display system. In XXII FISITA congress technical papers: Automotive systems technology: The future (pp. 2,6612,675). Warrendale, PA: Society of Automotive Engineers. 